\title{
ELECTRON SINGLE BUNCH ACCELERATION FROM LASER-PLASMA AT THE UNIVERSITY OF TOKYO
}

\author{
Nasr Hafz", R. Hemker, T. Kobayashi, K. Nakamura, H. Okuda, T. Ueda, K. Yoshii, T. Watanabe \\ M. Uesaka \\ Nuclear Engineering Research Laboratory, School of Engineering, University of Tokyo \\ 22-2 Shirane- Shirakata, Tokai, Naka, Ibaraki, 319-1188 Japan.
}

\begin{abstract}
The generation of an ultrashort relativistic electron bunch from a strong laser-plasma interaction have been studied by particle-in-cell simulations and observed experimentally. The electron beam was generated from the plasma created by focusing a Ti: sapphire 50fs 12 TW laser pulse at the edge of helium gas jet expanded into the vacuum from a pulsed supersonic nozzle.
\end{abstract}

\section{INTRODUCTION}

The rapidly increasing and outstanding advances in producing $\mathrm{T}^{3}$ - (Table-Top-Terawatt) laser systems motivated the extensive work in the field of laser-plasma based accelerators [1]. Of particular interest, is the standard scheme of the laser wake field accelerator (LWFA), in which an ultrashort, intense laser pulse propagates through an underdense plasma generating large amplitude plasma waves (wakefields) by the effect of the ponderomotive force associated with the laser pulse envelope $F_{p} \propto \nabla\left|E^{2}\right|$, where $|E|$ is the electric field of the laser pulse. The plasma wavelength (for density about $10^{18}-10^{19} \mathrm{~cm}^{-3}$ ) is of the order of $30-10 \mu \mathrm{m}$, therefore the longitudinal bunch length of the accelerated and/or injected (for further acceleration) electrons should be within this range. We proposed [2] such ultra-short electrons bunch generation by using a single ultra-short, ultra-intense laser pulse propagating in the plasma. So, the purpose of the present work is to generate relativistic (tens of MeV's) single electron bunch of duration in the sub-100fs range from the laser-plasma interaction. The basic idea for generating such electron bunch is to trap and accelerate some of the plasma electrons in a nonlinear plasma wave, which is forced to 'break' in the plasma. At the wavebreaking point, large amount of electrons are accelerated and leaving the plasma with high energies.

\section{TWO-DIMENSIONAL PIC SIMULATION}

\subsection{Simulation set-up}

The 2D simulations were done using the recently developed fully relativistic particle-in-cell code OSIRIS
[3]. The code is scalable parallel code and can be used for $2 \mathrm{D}$ as well as $3 \mathrm{D}$ simulations. It contains moving window algorithm that makes it possible to conduct simulations over the distances we have in our experiments with reasonable amount of computing resources. The simulation window, which was moving with the speed of light in the propagation direction of the laser pulse and thereby following the laser as well as any high-energy electrons, had in normalized units dimensions of $905 \mathrm{c} / \omega_{\mathrm{n}}$ in the $x_{1}$ direction and $2111 \mathrm{c} / \omega_{n}$ in the $x_{2}$ direction. $\omega_{n}$ is the frequency used for normalization. For convenience we choose $\omega_{\mathrm{n}}=\omega_{\mathrm{L}}$, the laser frequency. This normalization units corresponds to the length of the laser wavelength $\lambda_{\mathrm{L}}$ $=0.8 \mu \mathrm{m}$. The moving window size is therefore $115 \mu \mathrm{m} \times$ $269 \mu \mathrm{m}$ in MKSA units. The computational grid of the simulations was $2048 \times 768$ with four particles per cell. The simulations with the gas jet of $1.5 \mathrm{~mm}$ diameter were run for a time of $12600 \omega_{L}^{2}$, corresponding to a propagation distance of $1.6 \mathrm{~mm}$. The plasma density profile can be approximately described by a trapezoidal function. Within $500 \mu \mathrm{m}$ the plasma density ramps from zero (vacuum) up to $5 \times 10^{19} / \mathrm{cm}^{3}$. The density was kept constant at this value in about $500 \mu \mathrm{m}$. At last the density ramped down to zero in $500 \mu \mathrm{m}$. In the simulations we set the laser pulse as $12 \mathrm{TW} 50 \mathrm{fs}$ with spot size of $10 \mu \mathrm{m}$, initialized in vacuum and propagated through the plasma.

\subsection{Simulation results}

Figure (1) shows the simulation results. Figure (1a) and (1b) show the longitudinal phase-space of plasma electrons trapped and accelerated up to1 $10 \mathrm{MeV}$ by the plasma waves. Such very high-energy electrons were trapped in the wave at the beginning of the interaction. In Fig. (1a), after the laser has propagated distance of $c t=$ $0.49 \mathrm{~mm}$, it is clearly seen that amounts of electrons with high energies were trapped. Now we discuss the electron injection mechanism in this case. In Ref. [4], the plasmawavebreaking mechanism for such injection has been proposed. The steep plasma density gradient is necessary for the purpose. If the gradient is too long as in our simulations, the time delay between the maximal intensity wakefield and injected electrons can reduce essentially the efficiency of acceleration. However for a laser pulse with relativistic intensity $\left(a=e E / m_{0} c \omega>1\right)$, there is

*nasr@tokai.t.u-tokyo.ac.jp 

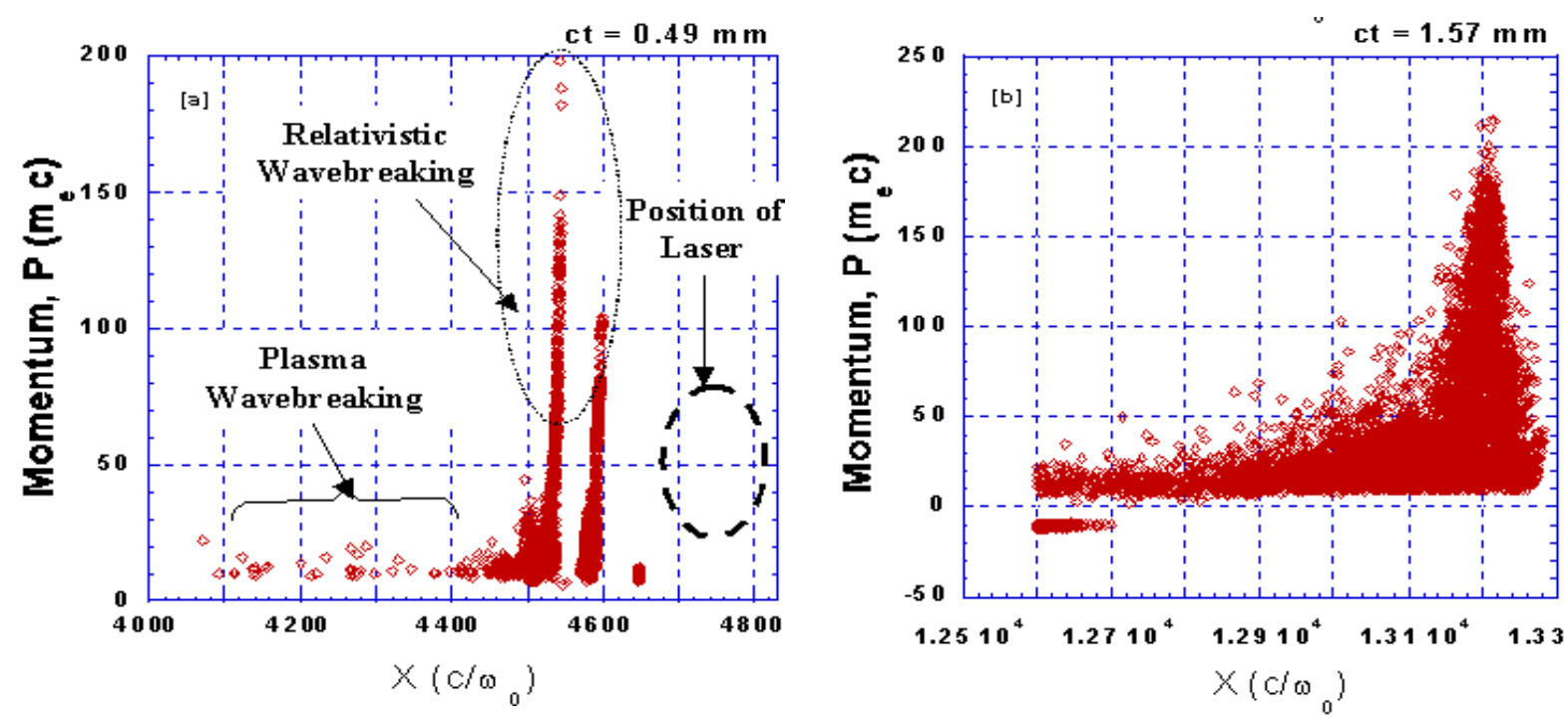

Figure 1: The simulation results, which show the longitudinal phase space of the trapped plasma electrons.

another mechanism for electron injection based on the breaking of waves produced by the relativistic acceleration of electrons into wakefield waves.

The effect of the ponderomotive force on electron acceleration decreases with the relativistic laser intensity because this force has $\gamma$ as a factor in denominator [5],

$$
\frac{d \vec{p}}{d t}=-\frac{m c^{2}}{2 \bar{\gamma}} \nabla \bar{a}^{2},
$$

where the bar means time averaging. With the laser intensity, this force grows as $a$ when $a>1$. In contrast to the ponderomotive force, the relativistic force, which acts along to the laser propagation direction, grows as $a^{2}$ [6]. The relativistic force creates waves with wavelengths of the order of the laser wavelength propagating with the laser pulse. The maximal energy of electrons in the waves is determined by the longitudinal component of the electron momentum,

$$
\gamma \sim p_{x}=a^{2} \sin ^{2}(\omega t+k x) / 2
$$

Since the wavelength of these waves is much shorter than the plasma wavelength, $\lambda_{\mathrm{p}}=2 \pi \mathrm{c} / \omega_{\mathrm{p}}$, the waves break into the wakefields behind the laser pulse producing energetic electrons. These electrons constitute the injection source for the following relativistic wave so that electrons acquire the maximal energy in the bunch just behind the laser pulse as shown in Fig. (1a)

The short wavelength waves appear behind the laser pulse and break into the wakefield with longer wavelength. This wavebreaking is an injection source for wake-field acceleration. These injection and acceleration mechanisms sustain as long as the laser pulse is relativistic and energetic. In this simulation, we observed that as the laser pulse propagates further through the plasma, it filaments, deteriorates and leaves the plasma in a distorted and diffracted shape. As a consequence, the ordering structure and energy of the relativistic wave and the electron trapping in it are all also deteriorated as in Fig. (1b). Disordering of the wave with the maximal energy electrons after the laser pulse deteriorated is a proof for the mechanism proposed.

Despite of the deterioration of the trapped electrons, they leave the plasma as shown in (Fig. 1b) in a single bunch with maximum energy of $110 \mathrm{MeV}$. The average energy of all electrons was $22.5 \mathrm{MeV}$. However, an energy spread of $100 \%$ was produced. Using a magnetic chicane, it seems possible to filter this beam in order to get a beam with lower energy spread. For example, in the energy range $82 \mathrm{MeV}-110 \mathrm{MeV}$, the bunch length was $3.5 \mu \mathrm{m}$ (equivalent to $\sim 10 f s$ ), the rms emittance $\sim 0.7 \mathrm{~mm}$ mrad and the total charge of accelerated electrons was about $580 \mathrm{pC}$.

\section{EXPERIMENTAL RESULTS}

The experimental set-up is shown in Fig. (2a) and described as follows: an OAP (off-axis parabolic) mirror focused the laser pulse at 1-3 mm above the top of a pulsed supersonic nozzle which produce a laminar flow of helium gas jet. The nozzle has a conic shape with a circular base of $2 \mathrm{~mm}$ in diameter. The maximum gas backing pressure was $\sim 80 \mathrm{~atm}$. The gas jet neutral density profile was measured by the Mach-Zender interferometry technique. Fig. (2b) shows the density distribution of helium gas generated from the jet at $80 \mathrm{~atm}$ backing pressure. The gas density at $3 \mathrm{~mm}$ above the jet is $\approx 1.38 \times 10^{20} \mathrm{~cm}^{-3}$. The density profile is Gaussian as shown in the figure, however constant density profile can be created by using Laval nozzle. The calculated laser spot size and intensity in vacuum above the nozzle were $12.6 \mu \mathrm{m}$ and $4 \times 10^{18} \mathrm{~W} / \mathrm{cm}^{2}$ respectively. The laser facility is based on the chirped-pulse amplification (CPA) technique at the wavelength of $0.8 \mu \mathrm{m}$, and is all-solid- 

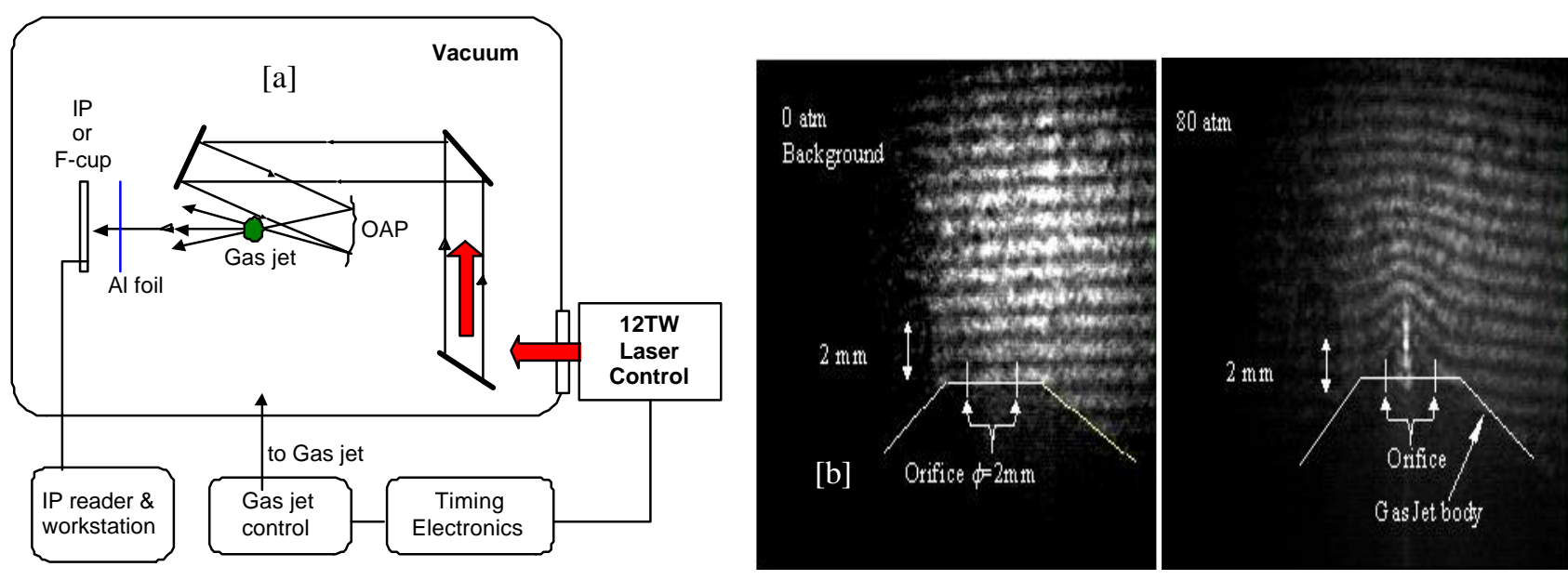

Figure 2: [a] The experimental set-up and [b] the gas density profile measured by Mach-Zender Interferometer.

state Ti: sapphire system, which provides pulses with peak power of $12 \mathrm{TW}$ with duration of $50 \mathrm{fs}$. The system consists of an oscillator system (FEMTOSOURCE-20) and a combined optical stretcher- amplifier- compressor system (ALPHA-10 S-12). The detailed operational procedures and performance of the laser facility are given in Ref. [2]. The transverse profiles of electron beams generated from helium plasma are shown in Fig. (3) for various gas pressures at the laser power of $4 \mathrm{TW}$. The gas pressures were 42, 52 and 62 atmospheres in Fig.3a-c, respectively. The profiles were detected by using an imaging plates (IP) inserted in the forward direction (i.e. same as the laser beam) inside the vacuum chamber at a distance of $200 \mathrm{~mm}$ from the focus point. Each electron image shown in Fig. (3) is an accumulation of about 200 pulses. The electron beams shown in Fig. (3a) and (3b) have approximately transverse Gaussian profile, and were emitted from the plasma in a cone-like shape with angles about $9^{\circ}$ and $7^{\circ}$ (at HWHM), respectively from the electron source. The beam profile was deviated from Gaussian at higher gas pressure in Fig (3c). In other measurement in the same experiment but at higher pressures and laser powers, we observed two-component spatial profile of the electron beam. Similar behavior has also been observed in previous experiments using longer laser pulses [7]. Using a Faraday Cup (FC) we performed a measurement of the beam charge outside of the vacuum chamber. The FC has been installed in the forward direction, $20 \mathrm{~cm}$ away from the interaction point. A vacuum flange made of Titanium with thickness about 20 $\mu \mathrm{m}$ was used so that the generated electrons can pass through it and measured outside the vacuum chamber. Using lead collimator in front of the FC then collimated the beam, in this configuration the FC was able to detect electron beam with divergence angle up to 0.07rad (i.e. $\left.4.2^{\circ}\right)$. In such measurement the average charge was about
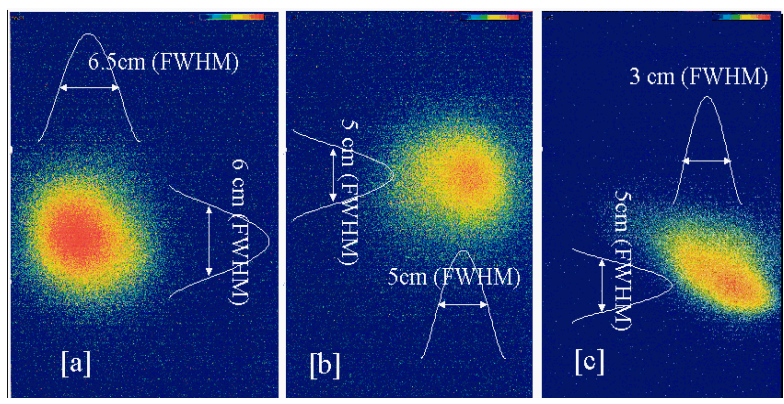

Figure 3: Electron beam profile at various gas pressures at $4 \mathrm{TW}$ laser power.

$15 \mathrm{pC}$ per pulse. We are planning to perform further experiments for fully diagnosing the electron beam, the laser pulse propagation, and the plasma diagnostics.

\section{REFERENCES}

[1] E. Esarey et al, IEEE Trans. Plasma Sci., PS-24, 252 (1996), and references therein.

[2] N. Hafz et al, Nucl. Instr. Meth., vol. A455, 148, (2000).

[3] R. Hemker, Ph.D. Thesis, University of California, Los Angeles, (2000).

[4] S. Bulanov et al., Phys. Rev. E 58, R5257-R5260. (1998).

[5] B. Quesnel and P. Mora, Phys. Rev. E 58, 3719 (1998).

[6] A. Zhidkov, A. Sasaki, T. Utsumi et al., Phys. Rev. E 62, 7232 (2000).

[7] S.Y. Chen et al., Phys. Plasmas 6, (1999) 4739-4749. 